\title{
A New Automated Method for Fast and Reliable Tilt-Series Acquisition in Electron Tomography
}

\author{
C. Kübel*, W.F. Voorhout, A.G. van Balen, D. Hubert, and M.T. Otten \\ * FEI Company, Application Laboratory, P.O. Box 80066, 5600 KA Eindhoven, The Netherlands
}

Electron tomography is a widely recognized method for obtaining high-resolution threedimensional images by electron microscopy (Figure 1) [1]. A sample is tilted around a single axis over a wide angular range $\left( \pm 70^{\circ}\right)$ with electron micrographs obtained at small angular tilt increments. Subsequently, the images of the tilt series are aligned and a reconstruction of the threedimensional volume is calculated. Traditionally, the acquisition of the tilt series has been timeconsuming and cumbersome, thus limiting the wide application of electron tomography in life science. A key to facilitate electron tomography is the recording of a tilt series in a fast and reliable way with minimal shifts and focus changes.

The electron tomography software on the Tecnai ${ }^{\mathrm{TM}} \mathrm{G}^{2}$ Sphera combines two novel approaches to meet these demands [2]. This software automatically determines the optimum acquisition position, and calibrates and stores the behavior of the holder (image shift and focus changes) at this optimized position. The stored holder-specific calibration (Figure 2) increases the speed and accuracy of the tilt series acquisition [3]. The reliability of the holder calibration depends on the reproducibility of the starting conditions, which is high due to new routines for automated focusing and eucentric-height determination.

In addition to the high degree of automation, which allows acquisition of a full tomography series of 141 pictures in about 25 minutes, the fully embedded Tecnai tomography software maintains a flexible approach for the user with state-of-the-art features. For example, on-line tracking can be performed optionally after the image acquisition using the tomography images themselves or before the image acquisition using low-resolution tracking images; important for cryo electron tomography, all procedures are now fully compatible with low-dose techniques. Furthermore, the software contains extensive documentation in form of on-line help and safeguards for detecting fault situations.

Current developments at FEI Company aim at extending the electron tomography methodology towards materials science, IC applications, and nano-technology. We are exploring the use of TEM, DF-STEM, and HAADF-STEM tomography for 3D visualization of the inorganic materials. HAADF-STEM imaging is a promising approach for acquiring a tomography tilt series as it minimizes diffraction effects in crystalline materials and enhances the difference between heavy and light elements [4].

\section{References}

[1] J. Frank, Three-Dimensional Electron Microscopy of Macromolecular Assemblies, Academic Press, San Diego, 1996.

[2] Visit the FEI booth for a demonstration of the Tecnai ${ }^{\mathrm{TM}} \mathrm{G} 2$ Sphera for fast and accurate automated TEM tomography. 
[3] U. Ziese et al., J. of Microscopy, in press.

[4] P. Midgley et al., Chem. Commun. (2001) 907.

[5] Courtesy of J.L. Murk, M.J. Kleijmeer, M.N. Lebbink (Department of Cell Biology and University Medical Center Utrecht, The Netherlands) and U. Ziese, A.J. Koster (Department of Molecular Cell Biology, Utrecht University, the Netherlands).
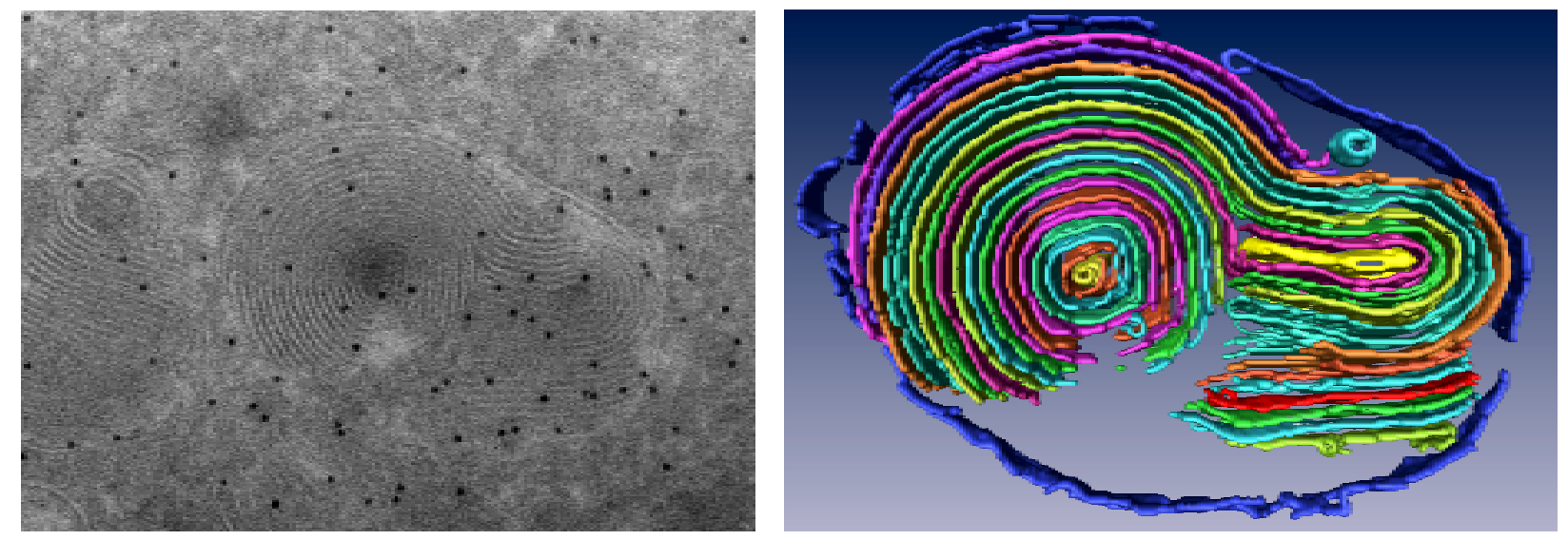

FIG 1. TEM image of lysosome in a $100 \mathrm{~nm}$ thick cryo section (reference image at $0^{\circ}$ tilt) and representation of the 3D structure after automatic recording of TEM tilt series and reconstruction of the dual axis data set [5].

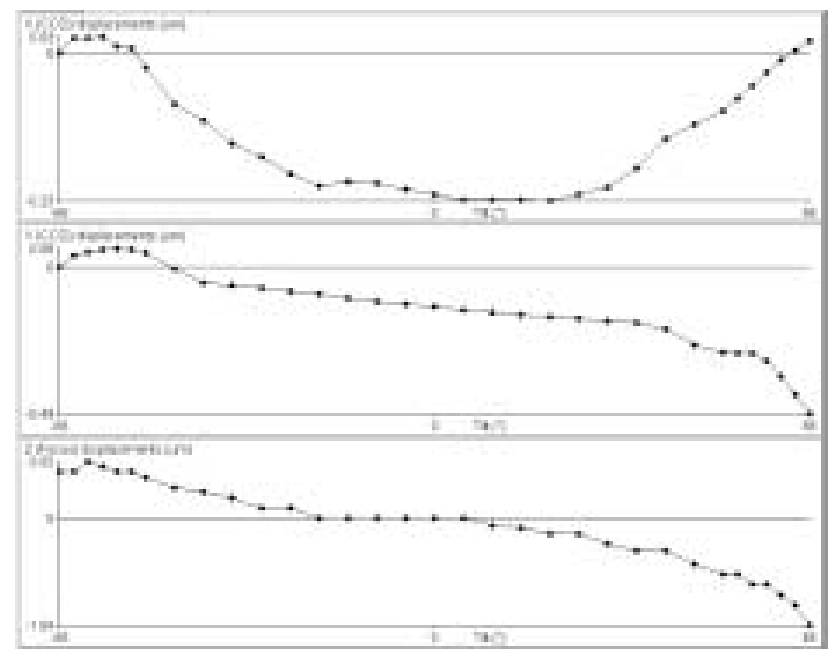

FIG 2: Example of a sample holder calibration file, which shows image shifts in x-direction of less than $300 \mathrm{~nm}$ and focal changes of less than $1.6 \mu \mathrm{m}$. 